\title{
Artificial Intelligence In Diagnosing And Treatment Of Oral Mucosal Lesions - A Systematic Review
}

\author{
Review Article
}

\section{Chenchulakshmi $\mathrm{G}^{1}$, M. Arvind ${ }^{2 *}$}

${ }^{1}$ Post graduate Resident, Department of Oral Medicine and Radiology, SIMATS, Chennai, 600007, India.

${ }^{2}$ Professor and Head of the Department of Oral Medicine and Radiology, SIMATS, Chennai, 600007, India.

\section{Abstract}

Background: Artificial intelligence (AI) has made deep inroads into dentistry in the last few years. In the modern-day world, artificial intelligence refers to any machine or technology that is able to mimic human cognitive skills like problem solving. To understand AI, it is important to know few of these key aspects. Artificial intelligence is termed as a capability of machines that exhibits a form of its own intelligence. Its aim was to develop machines that can learn through data so that they can solve the problems. Machine learning is part of AI, which depends on algorithms to predict outcomes based on a dataset. The purpose of machine learning is to facilitate machines to learn from data so they can resolve issues without human input. Neural networks are a set of algorithms that compute signals via artificial neurons. The purpose of neural networks is to create neural networks that function like the human brain. Deep learning is a component of machine learning that utilizes the network with different computational layers in a deep neural network to analyse the input data. The purpose of deep learning is to construct a neural network that automatically identifies patterns to improve feature detection. The aim of this systematic review was to analyze whether artificial intelligence is helpful in diagnosis and management of oral mucosal lesions.

Aim: This systematic review was to analyze whether artificial intelligence is helpful in diagnosis and management of oral mucosal lesions.

Search Methods: Electronic search of the following database was performed: PubMed, Cochrane central register of controlled trials, Google scholar and hand search.

Selection Criteria: According to PICO (Population, Intervention, Comparison, Outcome) criteria, the inclusion criteria were worked out. Articles including diagnosis and management of oral mucosal lesion were included.Articles that are related to non-AI areas, articles that are not written in English and articles not related to diagnosis and management of mucosal lesions were excluded.

Data Collection And Analysis: We used standard methodological procedures for selection of studies and collecting data. Risk of bias was evaluated and findings were synthesized.

Main Results: A total of 3 articles were included in this review that consisted of 3 Case-control studies.

Conclusion: Currently there is limited evidence to support application of artificial intelligence in diagnosing and managing oral mucosal lesions. Limited evidence available show artificial intelligence methods comparable to current, conventional diagnostic methods. Large multicentric data is required for integration of these methods into the digital workflow.

Keywords: Artificial Intelligence; Artificial Neural Networks; Oral Mucosal Lesions; Red And White Lesions Of Oral Cavity; Diagnosis And Management And Maxillofacial Lesions.

PICO: P -Oral mucosal lesions; I-Artificial Intelligence; C-Expert opinions, reference standards; 0-Effectiveness of diagnosis (sensitivity and specificity)

\section{Introduction}

Advancement in digitized technology for clinical examination has rendered health care data collection from dental patients less complex and cumbersome. Personalized dentistry requires taking mas- sive data sets into consideration for each patient. Conventional statistical analytics relies on specific assumptions and handcrafted markers, making it impractical in dealing with such high-volume data [1]. Artificial intelligence can be used as a useful modality in diagnosis and treatment of lesions of oral cavity and can be

*Corresponding Author:

M. Arvind,

Professor and Head of the Department of Oral Medicine and Radiology, SIMATS, Chennai, 600007, India.

E-mail: arvindmuthukrishnan@yahoo.com

Received: April 25, 2021

Accepted: August 30, 202

Published: September 05, 2021

Citation: Chenchulakshmi G, M. Arvind. Artificial Intelligence In Diagnosing And Treatment Of Oral Mucosal Lesions - A Systematic Review. Int J Dentistry Oral Sci. 2021;8(9):43024307. doi: http://dx.doi.org/10.19070/2377-8075-21000876

Copyright: M. Arvind ${ }^{\circ} 2021$. This is an open-access article distributed under the terms of the Creative Commons Attribution License, which permits unrestricted use, distribution and reproduction in any medium, provided the original author and source are credited. 
employed in screening and classifying suspicious altered mucosa undergoing premalignant and malignant changes.Artificial intelligence might accurately predict a genetic predisposition for oral cancer for a large population. The use of ANNs in the diagnoses subtypes of temporomandibular disorders has been studied by Bas B et al. They suggested that ANNs may act as an adjuvant diagnostic tool for dentist Artificial intelligence (AI) intends to reproduce the cognitive process of humans and can achieve the same outcome as medical professionals within a much shorter time frame. It excels in extracting information from historical data and benefits physicians by automating time-consuming tasks. Although the current development of AI is preliminary and medical tasks that contemporary AI can complete can almost be performed by humans, the emergence of $\mathrm{AI}$ in dentistry heralds an era of new technology with the potential to in which dental clinical care can be practiced efficiently.

Over the decades, new equipment was emerged in medical field, and we have witnessed the importance of medical imaging such as computed tomography, magnetic resonance imaging, ultrasound, mammography and X-ray and their contribution in successful diagnosis and treatment of various diseases.[2] With substantial increase in workload and complexity of work, potential fatigue of doctors, human experts and researchers may compromise the outcome.[2] Advanced breakthroughs in image recognition introduced by deep learning techniques, and media statements by researchers have portrayed artificial intelligence as the cause of demise of radiologists. However, the complex work performed by radiologists includes many other tasks that require common sense and general intelligence for problem solving tasks that cannot be achieved through AI. Understanding a case requires multiple basic medical and clinical specialities to provide plausible explanations for imaging findings. Also, advanced imaging modalities necessitate specialized intelligence for detection of anomalies, segmentation, and image classification [2]. Artificial intelligence (AI) is a technology, which has shifted from science fable into reality in the radiology practice in the last two decades [3]. Allan Turner one of the founders of AI defined it as the ability to achieve human-level performance in cognitive tasks by computers [4]. Implementation of $\mathrm{AI}$ in radiology is anticipated to significantly revolutionize the quality, value, and depth of radiology's contribution to patient care and population health, and radiologists work flow in next decade [5]. This makes it imperative that a radiologist be aware of $\mathrm{AI}$ and its applications in their field.

\section{Artificial Intelligence}

$\mathrm{AI}$ is a branch of computer science dedicated to the development of computer algorithms to accomplish tasks traditionally associ- ated with human intelligence, such as the ability to learn and solve problems [1]. This includes machine learning (ML), representation learning and deep learning. This systematic review was to done to analyze whether artificial intelligence is helpful in diagnosis and management of oral mucosal lesions.

\section{Machine learning (ML)}

Is a part of research on $\mathrm{AI}$ that seeks to provide knowledge to computers through data and observations without being explicitly programmed [6]. This allows a computer to correctly generalise a setting by tuning of parameters within the algorithm to optimize the goodness of fit between the input (ie, text, image, or video data fed into the algorithm) and output (ie, classification). For example, for a ML algorithm can detect a lymph node in head and neck image as normal or abnormal provided it is trained Radiologist by analysing thousands of such images which are labelled as normal or abnormal [1]. To sum it up ML algorithms are trained to give a specific answer by evaluating or learning a large number of exams that have been hand-labelled.

\section{Representation Learning}

Is a subtype of $\mathrm{ML}$ in which the computer algorithm learns the features required to classify the provided data. This does not require a hand labelled data like ML [7].

\section{Deep Learning}

Is a subfield of representation learning relying on multiple processing layers (hence, deep) to learn representations of data with multiple layers of abstraction. This algorithm uses multiple layers to detect simple features like line, edge and texture to complex shapes, lesions, or whole organs in a hierarchical structure. Basis of any radiologic interpretation is logical elimination of possible diagnosis. In this context, deep learning can potentially excel by learning a hierarchical normal representation of a specific type of image from a large number of normal exams [8]. This review was done to analyze whether artificial intelligence is helpful in diagnosing and management of oral mucosal lesions.

\section{Materials And Methods}

\section{Sources used}

An electronic search was done in PubMed, Cochrane library, Google scholar until 5th of March 2021. We used the complete search terms for PubMed, Cochrane. All electronic strategies had similar Title/Abstract and MeSH terms and texts.

\section{Search methodology \\ Details of PubMed Search}

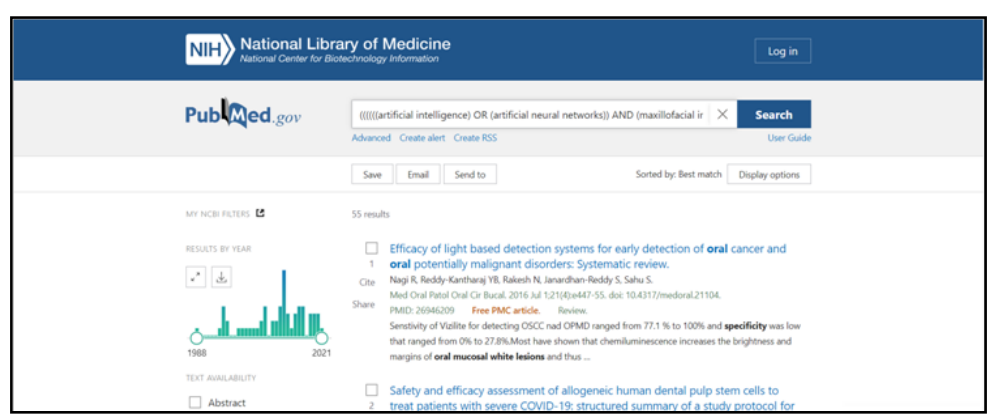



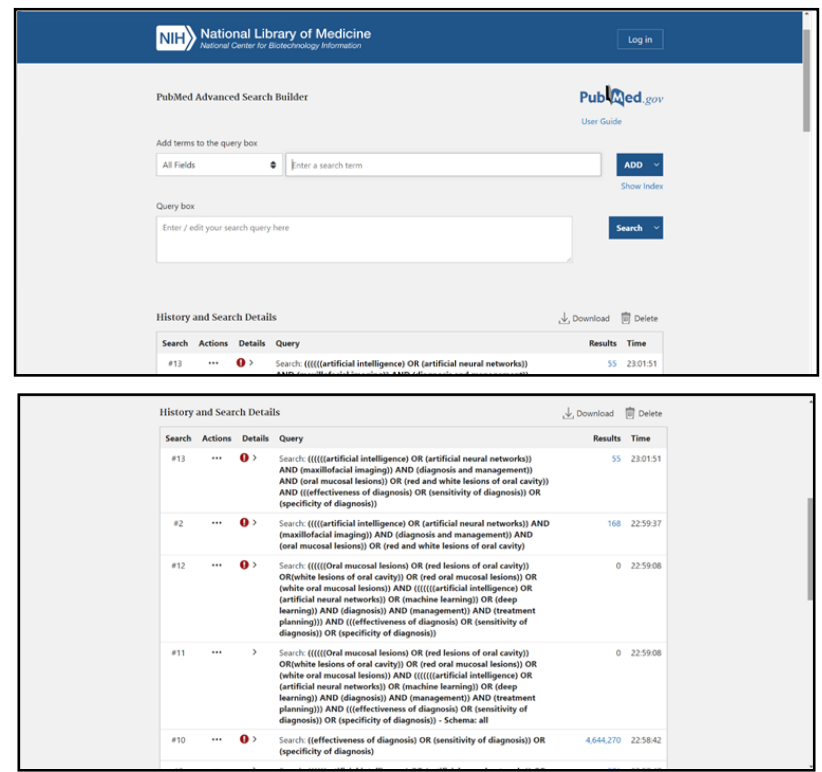

Details of Cochrane search
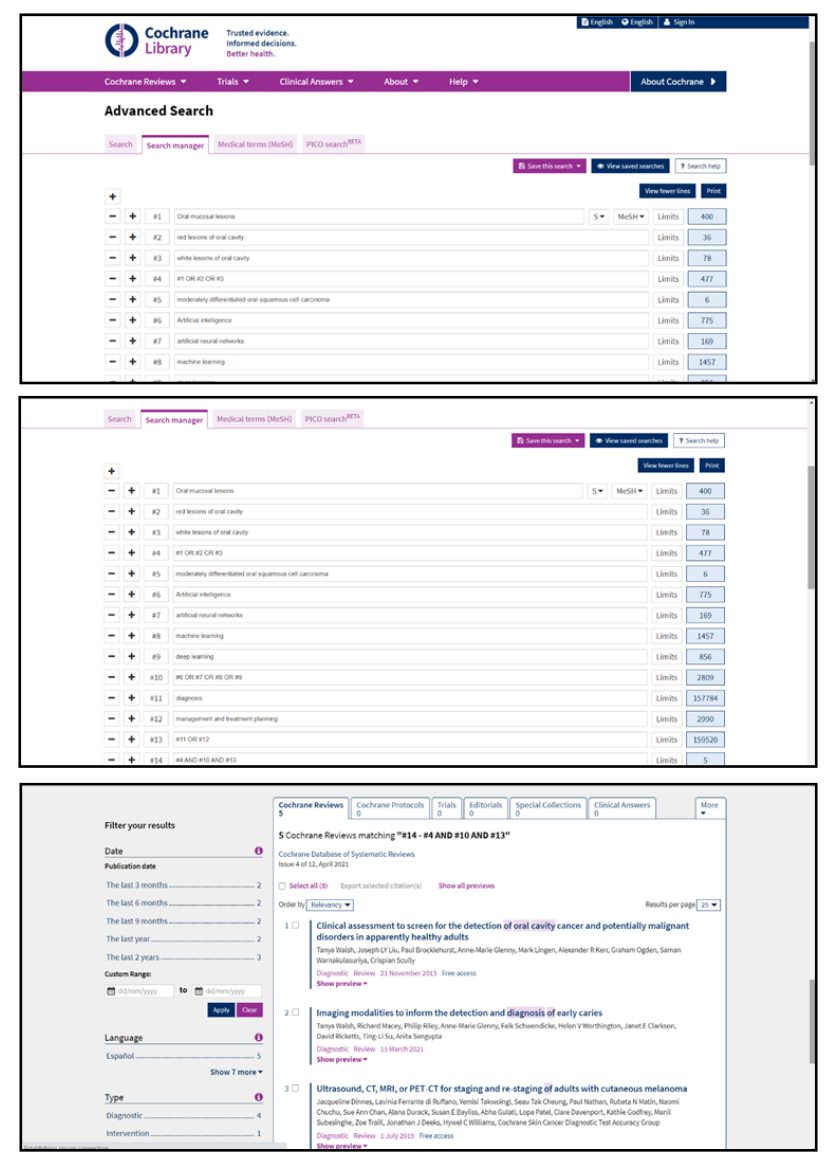

Details of Google scholar search

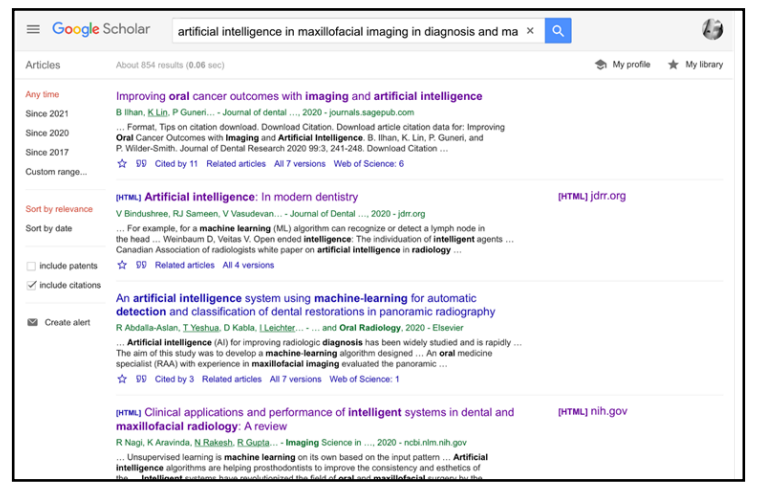




\section{Eligibility Criteria}

\section{Inclusion Criteria}

-Articles including diagnosis and management of oral mucosal lesions.

\section{Exclusion Criteria}

-Articles that are related to non-AI were excluded.

-Articles that are not written in English was excluded.

-Articles not related to diagnosis and management of oral mucosal lesions.

\section{Data Collection And Analysis}

Search and study selection was done by primary author and reviewed by second author. After initial search, potentially eligible articles were selected based on title and abstract. Full text of se- lected articles was reviewed and 3 relevant studies were identified and included in this systematic review.

\section{Variables Of Interest}

1) Effectiveness of diagnosis.

\section{Results}

The AHRQ classifies studies in seven levels according to the level of evidence: I) systematic review or meta-analysis; II) randomized controlled trials; III) controlled trials without randomization; IV) case-control studies and cohort studies; V) systematic reviews of descriptive and qualitative studies; VI) single descriptive or qualitative study; and VII) opinion of authorities and/or report of expert opinion.

\section{Quality Assessment Of The Studies}

Table 3 and 4 .

Search flowchart

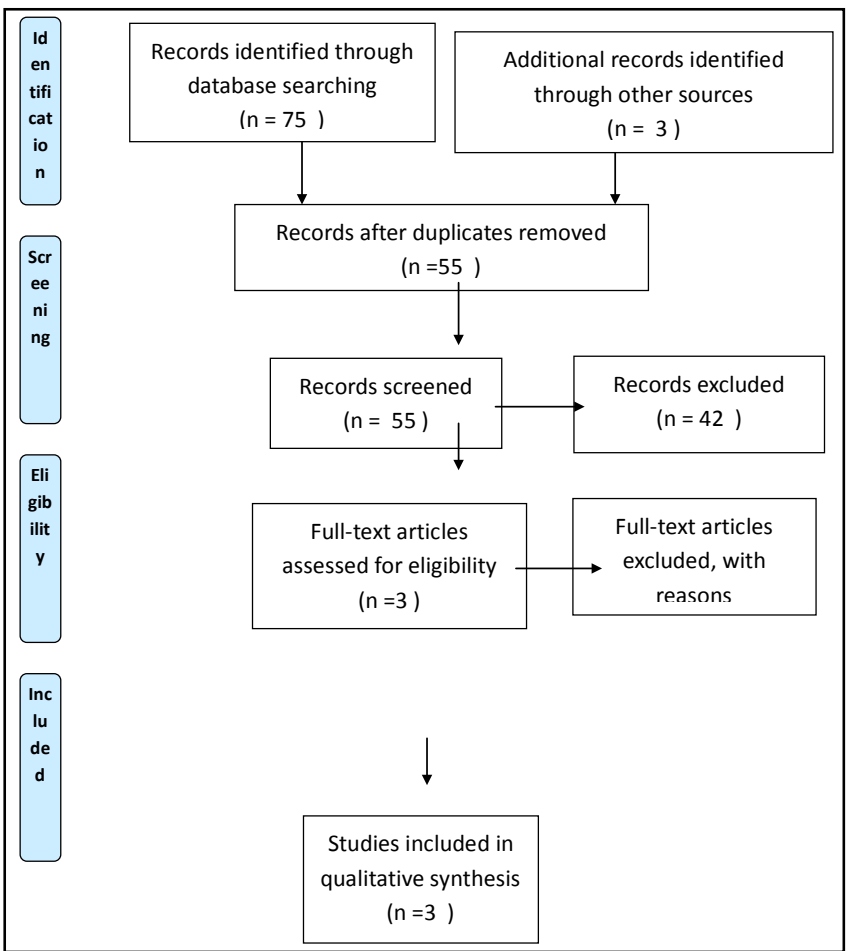

Table 1. General information of Selected studies.

\begin{tabular}{|c|c|c|c|c|c|c|c|}
\hline S . No & Author & Year & Sample size & Segmentation methods used & Sensitivity & Specificity & Accuracy \\
\hline 1 & Krishnan et al & 2009 & 158 & Neural network & $94.50 \%$ & $98.80 \%$ & Not given \\
\hline 2 & Rahman et al & 2018 & 216 & Support vector machine & - & - & $100 \%$ \\
\hline 3 & Krishnan et al & 2012 & 20 & Support vector machine & $90.46 \%$ & $87.54 \%$ & Not given \\
\hline
\end{tabular}

Table 2. Evidence Level Of Included Studies.

\begin{tabular}{|c|c|c|c|c|}
\hline S.NO & AUTHOR & YEAR & STUDY DESIGN & LEVEL OF EVIDENCE \\
\hline 1 & Krishnan et al & 2009 & Case control study & IV \\
\hline 2 & Krishnan et al & 2012 & Case control study & IV \\
\hline 3 & Rahman et al & 2018 & Case control study & IV \\
\hline
\end{tabular}


Table 3. Qualitative analysis of case-control studies done by Newcastle Ottawa scale.

\begin{tabular}{|c|c|c|c|c|c|c|c|c|c|}
\hline \multirow[t]{2}{*}{$\begin{array}{c}\text { Author/ } \\
\text { Year }\end{array}$} & & \multicolumn{3}{|c|}{ Selection } & \multirow{2}{*}{$\begin{array}{c}\begin{array}{c}\text { Compara- } \\
\text { bility }\end{array} \\
\begin{array}{c}\text { Based on } \\
\text { design of } \\
\text { analysis }\end{array}\end{array}$} & \multicolumn{3}{|c|}{ Exposure } & \multirow[t]{2}{*}{$\begin{array}{l}\text { Total (9 } \\
\text { points) }\end{array}$} \\
\hline & $\begin{array}{c}\text { Case } \\
\text { defini- } \\
\text { tion }\end{array}$ & $\begin{array}{l}\text { Repre- } \\
\text { sentation } \\
\text { of the } \\
\text { cases }\end{array}$ & $\begin{array}{l}\text { Selection } \\
\text { of con- } \\
\text { trols }\end{array}$ & $\begin{array}{l}\text { Defini- } \\
\text { tion of } \\
\text { controls }\end{array}$ & & $\begin{array}{l}\text { Ascer- } \\
\text { tainment } \\
\text { of expo- } \\
\text { sure }\end{array}$ & $\begin{array}{l}\text { Same method of } \\
\text { ascertainment for } \\
\text { cases and controls }\end{array}$ & $\begin{array}{l}\text { Non-re- } \\
\text { sponse } \\
\text { rate }\end{array}$ & \\
\hline $\begin{array}{l}\text { Krishnan } \\
\text { et al,2009 }\end{array}$ & $*$ & * & * & * & - & * & - & $*$ & 6 \\
\hline $\begin{array}{l}\text { Krishnan } \\
\text { et al, } 2012 \\
\end{array}$ & * & * & $*$ & - & - & - & - & $*$ & 4 \\
\hline $\begin{array}{c}\text { Rahman } \\
\text { et al,2018 }\end{array}$ & * & $*$ & $*$ & - & * & $*$ & - & - & 5 \\
\hline
\end{tabular}

Table 4. List Of Excluded Studies.

\begin{tabular}{|c|c|c|c|}
\hline S. No & AUTHOR & TITLE & REASON FOR EXCLUSION \\
\hline 1 & Aslan et al & $\begin{array}{c}\text { An artificial intelligence system using machine learning } \\
\text { for automatic detection and classification of dental } \\
\text { restorations in panoramic radiography }\end{array}$ & Not related to inclusion criteria \\
\hline 2 & Hung et al & $\begin{array}{c}\text { Current applications, Opportunities,and Limitations of } \\
\text { AI for 3D imaging in dental research and practice }\end{array}$ & Review article \\
\hline 3 & Nagi et al & $\begin{array}{c}\text { Clinical applications and performance of intelligent } \\
\text { systems in dental and maxillofacial radiology: A review }\end{array}$ & Review article \\
\hline 4 & Mudrak et al & $\begin{array}{c}\text { Artificial Intelligence and Deep learning in Dental Ra- } \\
\text { diology: A way forward in point of care radiology }\end{array}$ & Literature review \\
\hline 5 & Yaji et al & Artificial Intelligence in Dentomaxillofacial radiology & Not related to inclusion criteria \\
\hline
\end{tabular}

\section{Discussion}

AI is modernizing the traditional aspects of dentistry. AI based systems are often used for designing automated software programs that streamlines the diagnosis and data management in dentistry [9]. Mostly they are clinical decision support systems that assist and guide experts to make better decisions. These systems have been used for better diagnosis, treatment planning and also for predicting the prognosis [10]. The demand for these systems is booming due to their effectiveness in providing explanations and reasoning [11]. AI has revolutionized in the field of dentistry and making the dentist's task easier. The clinical decision support systems that work on the AI technology are mainly designed to provide expert support to the health professionals [12]. Clinical decision support systems is defined as, any computer program that has been designed to help health professionals in making clinical decisions, and also deals with the medical data or with the knowledge of medicine necessary for interpreting such data.

According to Krishnan et al, he used texture and the Otsu thresholding-based segmentation algorithms are applied to 158 sample images of size $1388 \times 1040$ pixels with $10 \times$ microscopic magnification. Therefore, the segmented class map (epithelium) obtained using these methods are compared with the common desired partition map (ground truth), which is marked independently by two oral oncologists to ensure region segmentation accuracy. Thus, the texture based segmentation yields $98 \%$ correctly extracted contours in most cases along with very good contour quality. He inferred that the Otsu's segmentation provides over-segmentation, because it assumes the histogram of the image is bimodal. The texture based segmentation results are shown for all three classes viz., normal, OSF without dysplasia and OSF with dysplasia. Moreover, the proposed methodology provides improved segmentation of the epithelial layer than that of the Otsu based segmentation method, because it combines both texture and intensity information of the epithelium. Otsu's method selects the threshold by minimizing the within class variance of the two groups of pixels separated by the thresholding operator. It does not depend on modelling the probability density functions, however, it assumes a bimodal distribution of gray-level values (i.e., if the image approximately fits this constraint, it will do a good job). However, the Otsu's method has the following disadvantages: (a) The method assumes that the histogram of the image is bimodal (i.e., two classes). (b) The method breaks down when the two classes are unequal (i.e., the classes have different sizes). (c) The two classes (object and background) are unequal; between class variance may have two maxima. (d) The selected threshold should correspond to a valley of the histogram. (e) The method does not work well with variable illumination. To overcome these limitations of Otsu's method, texture-based segmentation is performed for proper partitioning of pixels within regions. In doing this, we have considered the discontinuity in gray level, color, and texture at the interface as well as the intra-region similarity. However, the texture is different for the epithelial, subepithelial and background regions. The texture based segmentation algorithm is better able to separate these three regions through the use of texture features in addition to intensity. Here, the segmentation is unsupervised; the segmentation of oral pre-cancer images. 
In Rahman et al, Slides were collected from two local sources namely Ayursundra Healthcare Pvt. Ltd (Centre 1) and Dr. B Borooah Cancer Research Institute (Centre 2), (a Regional Cancer Centre recognised by the Government of India). A Leica ICC50 HD microscope was used to view and capture the images (size $2048 \times 1536)$. Images with $400 \times$ magnification were used for this study. 110 and 86 normal tissue images were captured from the slides collected from Centre 1 and Centre 2 respectively. Similarly, 113 and 88 malignant tissue images were captured from the slides collected from Centre 1 and Centre 2, respectively. Some images were defocussed during acquisition and became blurred. So, experienced and certified pathologists of the collaborating institutes rejected those images and selected the best consisting of 134 images with normal tissue and 135 images with malignant tissue for this study. They also marked the region of interest, which was used for ground truth preparation. The cropped images were used as inputs to the proposed system. As described, they were first subjected to the pre-processing step. It was observed that the quality of the images from the Centre 2, which were of poorer quality, were now improved and were suitable for proceeding to the next phase of feature extraction. There were some portions like the background, connective tissue region etc. on the collected images, which were not necessary for our study as we were dealing with the epithelium region only for normal tissue and cancerous region for malignant tissue. So, it was proposed to create a database with cropped images with the relevant portions from the collected images. At the time of image acquisition, the original size of all the images was $2048 \times 1536$ [13]. Thereafter, experienced and certified pathologists have selected the relevant portions, that is, region of interest (ROI), which was used for ground truth preparation. Depending upon the affected region, we have cropped matrices of various sizes from the original image. In some images, the ROI is big and in some images, it is small. These cropped images were considered as input to the proposed method. The size of the cropped images is not an affecting factor in the proposed method. While cropping, for some images, two to three images were created from one original image. The database with cropped images contains 223 images with normal tissue and 253 images with malignant tissue. The texture features of the images are considered for performing the classification. The approaches used for feature extraction are GLCM and histogram techniques. For classification, linear SVM has been used and the results prove to be very satisfactory as $100 \%$ accuracy is being achieved. It is hoped that it can be used as an efficient framework for developing computer-aided diagnostic tools, which would assist the clinicians/pathologists for rapid evaluation of screening of tumorous lesions from normal ones [14].

\section{Conclusion}

Currently there is limited evidence to support application of artificial intelligence in diagnosing and managing oral mucosal lesions. Limited evidence available show artificial intelligence methods comparable to current, conventional diagnostic methods. Large multicentric data is required for integration of these methods into the digital workflow.

\section{References}

[1]. Shen D, Wu G, Suk HI. Deep learning in medical image analysis. Annual review of biomedical engineering. 2017 Jun 21;19:221-48.

[2]. Tang A, Tam R, Cadrin-Chênevert A, Guest W, Chong J, Barfett J, et al. Canadian Association of Radiologists White Paper on Artificial Intelligence in Radiology. Can Assoc Radiol J. 2018 May;69(2):120-135. Pubmed PMID: 29655580.

[3]. Ng A. Should radiologists be worried about their jobs? Breaking news: We can now diagnose pneumonia from chest X-rays better than radiologists. 2017

[4]. Kooi T, Litjens G, van Ginneken B, Gubern-Mérida A, Sánchez CI, Mann $\mathrm{R}$, et al. Large scale deep learning for computer aided detection of mammographic lesions. Med Image Anal. 2017 Jan;35:303-312. Pubmed PMID: 27497072.

[5]. Drozdzal M, Chartrand G, Vorontsov E, Shakeri M, Di Jorio L, Tang A, et al. Learning normalized inputs for iterative estimation in medical image segmentation. Med Image Anal. 2018 Feb;44:1-13. Pubmed PMID: 29169029.

[6]. Becker AS, Marcon M, Ghafoor S, Wurnig MC, Frauenfelder T, Boss A. Deep Learning in Mammography: Diagnostic Accuracy of a Multipurpose Image Analysis Software in the Detection of Breast Cancer. Invest Radiol. 2017 Jul;52(7):434-440. Pubmed PMID: 28212138.

[7]. Ramesh AN, Kambhampati C, Monson JR, Drew PJ. Artificial intelligence in medicine. Annals of the Royal College of Surgeons of England. 2004 Sep;86(5):334.

[8]. Tang A, Tam R, Cadrin-Chênevert A, Guest W, Chong J, Barfett J, et al. Canadian Association of Radiologists White Paper on Artificial Intelligence in Radiology. Can Assoc Radiol J. 2018 May;69(2):120-135. Pubmed PMID: 29655580.

[9]. Faggella D. "What is machine learning? Tech emergence". (2017).

[10]. Schleyer TK, Thyvalikakath TP, Spallek H, Torres-Urquidy MH, Hernandez P, Yuhaniak J. Clinical computing in general dentistry. J Am Med Inform Assoc. 2006 May-Jun;13(3):344-52. Pubmed PMID: 16501177.

[11]. Mendonça EA. Clinical decision support systems: perspectives in dentistry. Journal of dental education. 2004 Jun;68(6):589-97.

[12]. Begum S, Ahmed MU, Funk P, Xiong N, Von Schéele B. A case-based decision support system for individual stress diagnosis using fuzzy similarity matching. Computational Intelligence. 2009 Aug;25(3):180-95.

[13]. Khanna S. Artificial intelligence: contemporary applications and future compass. Int Dent J. 2010 Aug;60(4):269-72. Pubmed PMID: 20949757.

[14]. Burges CJ. A tutorial on support vector machines for pattern recognition. Data mining and knowledge discovery. 1998 Jun;2(2):121-67.

[15]. Rahman TY, Mahanta LB, Chakraborty C, Das AK, Sarma JD. Textural pattern classification for oral squamous cell carcinoma. J Microsc. 2018 Jan;269(1):85-93. Pubmed PMID: 28768053. 